
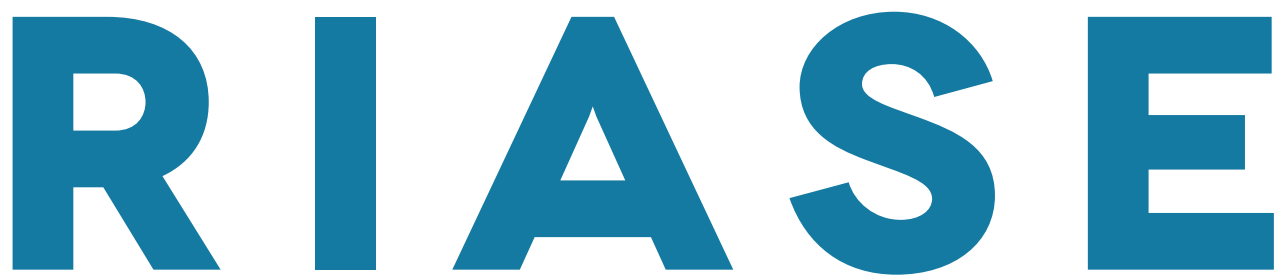

REVISTA IBERO-AMERICANA DE SAÚDE E ENVELHECIMENTO REVISTA IBERO-AMERICANA DE SALUD Y ENVEJECIMIENTO

\title{
CUIDADOS PRECOCES NA REABILITAÇÃO DA FUNCIONALIDADE EM DOENTES COM ACIDENTE VASCULAR CEREBRAL
}

\section{EARLY CARE IN FUNCTIONALITY REHABILITATION IN STROKE PATIENTS}

\section{ATENCIÓN TEMPRANA DE REHABILITACIÓN EN LA FUNCIONALIDAD CON ACCIDENTE CEREBROVASCULAR}

\footnotetext{
Maria Inês Guerreiro - Serviço de Formação Profissional da Unidade Local de Saúde do Litoral Alentejano - Centro de Saúde de Alcácer do Sal, Portugal.

ORCID: https://orcid.org/0000-0002-8112-7753

Maria José Abrantes Bule - Departamento de Enfermagem, Universidade de Évora, Évora, Portugal. ORCID: https://orcid.org/0000-0002-0511-2920

Gorete Mendonça dos Reis - Departamento de Enfermagem, Universidade de Évora; Comprehensive Health Research Center (CHRC), Évora, Portugal.

ORCID: https://orcid.org/0000-0003-1001-4142
} 


\section{RESUMO}

Objetivo: Analisar resultados da intervenção terapêutica precoce do enfermeiro de reabilitação, na funcionalidade, em doentes com acidente vascular cerebral.

Métodos: Estudos de caso múltiplos segundo as guidelines da CAse REport (CARE). Participaram quatro doentes, idade média de 71,8 anos ( $\mathrm{DP}=9,9$ anos). Estudo aprovado em Comissão de Ética para a Saúde.

Resultados: $\bigcirc$ treino de autocuidados e do equilíbrio conduziu três doentes ao score de dependência modificada (com assistência até $25 \%$ da atividade), um participante manteve o nível de dependência modificada (assistência até 50\% da atividade). A média de evolução nos autocuidados variou entre 0,75 e 1,75 pontos, evolução mais favorável na alimentação, higiene, vestir a metade superior e uso do sanitário. A evolução média no equilíbrio foi de 12,75 pontos, três doentes mantiveram-se na categoria Aceitável e um evoluiu para Bom.

Conclusão: Verificaram-se ganhos na funcionalidade, sensíveis aos cuidados de enfermagem de reabilitação.

Palavras-chave: Autocuidado; Enfermagem de Reabilitação; Exercício Terapêutico.

\section{ABSTRACT}

Objective: Analyze the results of the early therapeutic intervention of the rehabilitation nurse in functionality in stroke patients.

Methods: Multiple case studies according to CAse REport (CARE) guidelines. Four patients participated, mean age 71.8 years (SD=9.9 years). Study approved by the Ethics Committee for Health.

Results: Self-care and balance training led three patients to the modified dependency score (with assistance up to 25\% of activity), one participant maintained the modified dependency level (with assistance up to 50\% of activity). The average evolution in self-care ranged from 0.75 to 1.75 points, more favorable evolution in food, hygiene, dressing the upper half and use of the toilet. The mean evolution in balance was 12.75 points, three patients remained in the Acceptable category and one evolved to Good.

Conclusion: There were gains in functionality, sensitive to rehabilitation nursing care. Keywords: Exercise therapy; Rehabilitation Nursing; Self-Care. 


\section{RESUMEN}

Objetivo: Analizar los resultados de la intervención terapéutica temprana de la enfermera de rehabilitación, en la funcionalidad, en pacientes con accidente cerebrovascular.

Métodos: Estudios de casos múltiples de acuerdo con las pautas de CAse REport (CARE). Participaron cuatro pacientes, edad media 71,8 años ( $\mathrm{DP}=9,9$ años). Estudio aprobado por el Comité de Ética para la Salud.

Resultados: El autocuidado y el entrenamiento del equilibrio llevaron a tres pacientes a la puntuación de dependencia modificada (con asistencia de hasta el 25\% de la actividad), un participante mantuvo el nivel de dependencia modificada (asistencia de hasta el 50\% de la actividad). La evolución promedio en el autocuidado varió entre 0,75 y 1,75 puntos, una evolución más favorable en alimentación, higiene, vestir la parte superior del cuerpo y usar el baño. La evolución promedio en equilibrio fue de 12,75 puntos, tres pacientes permanecieron en la categoría Aceptable y uno evolucionó a Bueno.

Conclusión: Se confirman mejoras en la funcionalidad, sensibles a los cuidados de rehabilitación enfermera.

Descriptores: Autocuidado; Ejercicio Terapéutico; Enfermería de Rehabilitación.

\section{INTRODUÇÃO}

Os doentes que sobrevivem a um acidente vascular cerebral (AVC), apresentam frequentemente alterações da funcionalidade e graus de dependência variáveis. O AVC é uma das causas mais comuns de dependência a longo prazo(1-2).

Na revisão da literatura realizada por Aries \& Hunter ${ }^{(3)}$, relativa à intervenção de reabilitação em pessoas com AVC, 24 horas após o acontecimento, constatou-se que uma abordagem multidisciplinar com estratégias de reabilitação pode promover a recuperação destas pessoas, tais como: um ambiente enriquecido, com interação social, oportunidades de repetição e prática de movimentos e atividades funcionais envolvendo membros paréticos, estimulação sensorial e atividade precoce fora do leito. Concluíram os autores que a intervenção na pessoa com AVC deve ser promovida 24 horas após o acidente vascular, com ênfase na intervenção da equipa de enfermagem que está diretamente ligada aos cuidados e tem uma posição privilegiada para coordenar a abordagem e incentivar a pessoa com limitação física, de forma a otimizar a sua recuperação e sobretudo promover também o trabalho em equipa ${ }^{(3)}$. 
A European Stroke Organization (ESO) refere com evidência de grau B a mobilização precoce de moderada intensidade, em doentes com AVC e considera o treino progressivo de atividades da vida diária (Grau A)(4). A intensidade da mobilização precoce deve ser adaptada à situação clinica e monitorizada na perspetiva dos sinais e sintomas do doente (Grau C) $)^{(4)}$.

Os casos relatados referem-se a quatro doentes com AVC, não internados em Unidade diferenciada, com alterações da funcionalidade resultantes da hemiplegia e que foram sujeitos a cuidados de enfermagem de reabilitação com treino de atividades da vida diária (AVD) e treino do equilíbrio iniciados na fase aguda. A avaliação diagnóstica foi realizada com instrumentos validados, o protocolo de intervenção foi elaborado com base nas evidências e os resultados foram obtidos pela diferença ente a avaliação inicial e a avaliação final. Os cuidados de enfermagem de reabilitação foram eficazes na transição da dependência completa para dependência modificada.

Objetivo

Analisar resultados da intervenção terapêutica precoce do enfermeiro de reabilitação, na funcionalidade, em doentes com AVC.

\section{MÉTODO}

Desenho do estudo

Estudos de caso múltiplos segundo as guidelines da CAse REport(5) de modo a serem cumpridos os princípios de precisão e transparência dos resultados e do contributo destes para a enfermagem de reabilitação. O protocolo de intervenção seguiu a metodologia do processo de enfermagem e foi usada a Classificação Internacional para a prática de Enfermagem (CIPE), na versão 2. $0^{(6)}$. O estudo realizou-se no período de setembro de 2018 a janeiro de 2019, partindo da seguinte questão de investigação "quais os ganhos em saúde obtidos através da intervenção terapêutica precoce do enfermeiro de reabilitação, na funcionalidade, em doentes com acidente vascular cerebral?"

Princípios éticos

Estudo aprovado em Comissão de Ética para a Saúde da Unidade Local de Saúde. Foram respeitados os princípios da Declaração de Helsínquia, aos participantes foi apresentado o pedido de consentimento informado. Foi salvaguardado o princípio do livre abandono à participação. Em todas as fases de recolha, análise e divulgação de resultados foi mantido o anonimato dos participantes. 
Participantes

Doentes internados por AVC, na fase aguda, excluindo os casos que por alteração do nível de consciência não apresentavam manifestações de interação consciente com os profissionais e com o ambiente. Participaram quatro doentes que são adiante designados por A1 a A4. O tipo de amostragem foi por conveniência.

Protocolo de intervenção

O protocolo de intervenção foi elaborado a partir da revisão da literatura e estruturado segundo as etapas do processo de enfermagem:

Avaliação - Foram recolhidos dados através de um formulário, composto por parâmetros biofísicos. Para avaliação da funcionalidade foi aplicada a Medida de Independência Funcional (MIF), constituída por 18 itens distribuídos em seis domínios: Autocuidados; Controle de Esfíncteres, Mobilidade e Transferências, Locomoção, Comunicação e Consciência do Mundo Exterior. A pontuação máxima é de 126 pontos, que corresponde a independência completa e o mínimo de 18 pontos que representa dependência completa ${ }^{(7)}$. Podem ainda ser identificados 4 pontos de corte: 18 pontos (dependência completa); 19 - 60 pontos (dependência modificada com assistência até 50\% da tarefa); 61 - 103 pontos (dependência modificada, com assistência até 25\% da tarefa); 104 - 126 pontos (independência completa) $)^{(8)}$.

O equilíbrio foi avaliado pela Escala de Berg (EEB), foi traduzida e adaptada para a língua portuguesa, considerando-se a versão brasileira um instrumento confiável para ser usado na avaliação do equilíbrio(9). É composta por 14 itens onde estão incluídas atividades funcionais específicas em diferentes bases de apoio. Cada item apresenta uma escala ordinal de 5 alternativas que recebem uma pontuação de 0 a 4, num total de 56 pontos. As pontuações finais que variem entre 0 a 20 representam a diminuição do equilíbrio, entre 21 a 40 representam equilíbrio aceitável, e entre 41 a 56 representam um bom equilíbrio(8).

A análise dos dados de avaliação conduziu à formulação dos diagnósticos de enfermagem de reabilitação: Autocuidado arranjar-se comprometido; autocuidado uso do sanitário comprometido, autocuidado comer e beber comprometido; equilíbrio corporal comprometido e andar ou andar com auxiliar de marcha comprometido(8).

Plano de intervenção - Os cuidados de enfermagem de reabilitação foram planeados de acordo com os diagnósticos de enfermagem (Tabela 1). 
Tabela 1 - Plano de intervenção de enfermagem de reabilitação.

\begin{tabular}{|c|c|}
\hline Diagnóstico de Enfermagem & Intervenção \\
\hline \multirow[t]{4}{*}{ Autocuidado arranjar-se comprometido. } & Instruir autocuidado. \\
\hline & Demonstrar lavar, secar, vestir e despir. \\
\hline & Treinar lavar, secar, vestir, despir. \\
\hline & $\begin{array}{l}\text { Treinar a utilização de dispositivos } \\
\text { de apoio ao autocuidado. }\end{array}$ \\
\hline \multirow[t]{2}{*}{ Autocuidado uso do sanitário comprometido. } & Treino de hábitos de eliminação. \\
\hline & $\begin{array}{l}\text { Treinar a utilização de dispositivos para } \\
\text { eliminação. }\end{array}$ \\
\hline \multirow[t]{4}{*}{ Autocuidado comer e beber comprometido. } & Instruir sobre o autocuidado. \\
\hline & Treinar o autocuidado. \\
\hline & Reeducar membro superior dominante. \\
\hline & $\begin{array}{l}\text { Treinar utilização de dispositivos de apoio } \\
\text { para o autocuidado. }\end{array}$ \\
\hline Equilíbrio corporal comprometido. & $\begin{array}{l}\text { Treino de equilíbrio funcional, dinâmico } \\
\text { e estático. }\end{array}$ \\
\hline \multirow{2}{*}{$\begin{array}{l}\text { Andar comprometido/Andar com auxiliar } \\
\text { de marcha comprometido. }\end{array}$} & Treino de transferências pelo lado afetado. \\
\hline & Treino de marcha. \\
\hline
\end{tabular}

As intervenções foram realizadas em dias alternados, no período da manhã, ajustados os treinos aos horários das atividades habituais na unidade de internamento. O protocolo de cuidados foi realizado pelo mesmo profissional, sem recurso a outros elementos da equipa. Cada sessão totalizou em média 90 minutos distribuídos em diferentes momentos: Autocuidados de higiene, vestir e despir e utilização do sanitário; exercícios terapêuticos e um terceiro momento para treino de marcha. O plano foi adequado à evolução do doente, no sentido de aumentar a sua capacidade para executar as tarefas e foi concluído no momento da alta.

Avaliação de resultados - Os resultados sensíveis à intervenção de enfermagem de reabilitação foram extraídos pelas diferenças obtidas na avaliação inicial, em confronto com as de avaliação final. 


\section{RESULTADOS}

Os resultados apresentam-se tal como definido no protocolo CARE ${ }^{(5)}$ : Caracterização dos casos, dados clínicos, dados relevantes ocorridos durante a intervenção (i.e. linha do tempo), avaliação diagnóstica, resultados e discussão. Os quatro casos são apresentados separadamente e na discussão dos resultados salientam-se as diferenças e as semelhanças encontradas.

Apresentam-se os dados de caracterização biográfica relativos à idade e ao sexo e foram considerados relevantes os dados clínicos relativos ao diagnóstico médico de admissão, mas também os diagnósticos secundários ativos (Tabela 2).

Tabela 2 - Caracterização dos casos.

\begin{tabular}{|c|c|c|c|c|}
\hline & $\mathrm{A} 1$ & A2 & A3 & A4 \\
\hline Sexo & Masculino & Masculino & Feminino & Masculino \\
\hline Anos de idade & 69 & 82 & 57 & 79 \\
\hline $\begin{array}{l}\text { Diagnóstico } \\
\text { de admissão }\end{array}$ & $\begin{array}{c}\text { AVC } \\
\text { Hemorrágico } \\
\text { do núcleo } \\
\text { capsular direito } \\
\text { com inundação } \\
\text { tetra- } \\
\text {-ventricular }\end{array}$ & $\begin{array}{l}\text { AVC Isquémico } \\
\text { do núcleo } \\
\text { capsular e peri- } \\
\text {-ventricular } \\
\text { à direita }\end{array}$ & $\begin{array}{l}\text { AVC Isquémico } \\
\text { do hemisfério } \\
\text { cerebeloso } \\
\text { esquerdo }\end{array}$ & $\begin{array}{l}\text { AVC isquémico } \\
\text { (artéria cerebral } \\
\text { média esquerda) }\end{array}$ \\
\hline $\begin{array}{l}\text { Diagnósticos } \\
\text { secundários } \\
\text { ativos }\end{array}$ & $\begin{array}{c}\text { HTA } \\
\text { Obesidade AVC } \\
\text { hemorrágico } \\
\text { Desorientação } \\
\text { espaço/tempo }\end{array}$ & $\begin{array}{c}\text { HTA } \\
\text { Doença } \\
\text { cerebrovascular }\end{array}$ & $\begin{array}{l}\text { Status pós } \\
\text { prótese total } \\
\text { da anca. }\end{array}$ & \\
\hline
\end{tabular}

AVC - acidente vascular cerebral; HTA - hipertensão arterial.

Os défices iniciais apresentados pelos doentes alteraram-se e são relevantes, no âmbito do projeto de intervenção. Apresentam-se segundo uma linha cronológica, designada tal como previsto no protocolo CARE e reportam-se aos défices motores. O caso A1 apresentava diminuição da força muscular em ambos os membros inferiores, facto associado ao motivo de internamento, mas também aos diagnósticos secundários ativos. O caso A3 não apre- 
sentava alteração da força, coincidente com a localização da lesão. A hemiparesia direita verificou-se nos casos A2 e A4. A evolução da força muscular verificou-se em todos os casos nos quais foram registadas alterações. A avaliação inicial e final do tónus muscular pela Escala de Ashworth Modificada documenta a inexistência de alterações (Tabela 3).

Tabela 3 - Cronologia de dados relevantes.

\begin{tabular}{|c|c|c|c|c|c|c|c|c|}
\hline \multirow[b]{2}{*}{ Tempo } & \multicolumn{2}{|c|}{ A1 } & \multicolumn{2}{|c|}{$\mathrm{A} 2$} & \multicolumn{2}{|c|}{ A3 } & \multicolumn{2}{|c|}{ A4 } \\
\hline & Início & Fim & Início & Fim & Início & Fim & Início & Fim \\
\hline & \multicolumn{8}{|c|}{ Força Muscular - Medical Research Council Muscle Scale } \\
\hline Membro superior direito & $5 / 5$ & $5 / 5$ & $3 / 5$ & $4 / 5$ & $5 / 5$ & $5 / 5$ & $4 / 5$ & $5 / 5$ \\
\hline Membro superior esquerdo & $5 / 5$ & $5 / 5$ & $5 / 5$ & $5 / 5$ & $5 / 5$ & $5 / 5$ & $5 / 5$ & $5 / 5$ \\
\hline Membro inferior direito & $3 / 5$ & $4 / 5$ & $4 / 5$ & $5 / 5$ & $5 / 5$ & $5 / 5$ & $4 / 5$ & $5 / 5$ \\
\hline \multirow[t]{2}{*}{ Membro inferior esquerdo } & $3 / 5$ & $4 / 5$ & $5 / 5$ & $5 / 5$ & $5 / 5$ & $5 / 5$ & $5 / 5$ & $5 / 5$ \\
\hline & \multicolumn{8}{|c|}{ Tónus Muscular - Escala de Ashworth modificada } \\
\hline Membro superior direito & \multicolumn{2}{|c|}{0} & \multicolumn{2}{|c|}{0} & \multicolumn{2}{|c|}{0} & \multicolumn{2}{|c|}{0} \\
\hline Membro superior direito & \multicolumn{2}{|c|}{0} & \multicolumn{2}{|c|}{0} & \multicolumn{2}{|c|}{0} & \multicolumn{2}{|c|}{0} \\
\hline Membro superior esquerdo & \multicolumn{2}{|c|}{0} & \multicolumn{2}{|c|}{0} & \multicolumn{2}{|c|}{0} & \multicolumn{2}{|c|}{0} \\
\hline Membro inferior direito & \multicolumn{2}{|c|}{0} & \multicolumn{2}{|c|}{0} & \multicolumn{2}{|c|}{0} & \multicolumn{2}{|c|}{0} \\
\hline
\end{tabular}

A funcionalidade e o equilíbrio foram os parâmetros usados na avaliação diagnóstica e de resultados. Todos os participantes diminuíram o seu nível de dependência inicial, com o aumento do score da MIF que variou entre 13 a 20 pontos (média M=17; desvio padrão $\mathrm{DP}=3,08)$. De acordo com os pontos de corte da MIF a maioria dos participantes atingiu um score de classificação de dependência modificada, com assistência até 25\% da atividade. Apenas o participante A1 manteve o nível de dependência modificada, com assistência até $50 \%$ da atividade (Tabela 4 ). 
Tabela 4 - Avaliação da funcionalidade pré e pós intervenção de enfermagem de reabilitação.

\begin{tabular}{|c|c|c|c|c|c|c|c|c|}
\hline \multirow[b]{2}{*}{ MIF } & \multicolumn{2}{|c|}{ A1 } & \multicolumn{2}{|c|}{ A2 } & \multicolumn{2}{|c|}{ A3 } & \multicolumn{2}{|c|}{ A4 } \\
\hline & Início & Fim & Início & Fim & Início & Fim & Início & Fim \\
\hline & \multicolumn{8}{|c|}{ Autocuidados } \\
\hline Alimentação & 4 & 5 & 4 & 6 & 5 & 7 & 5 & 7 \\
\hline Higiene & 2 & 3 & 2 & 4 & 4 & 7 & 3 & 4 \\
\hline Banho & 2 & 3 & 2 & 3 & 4 & 4 & 3 & 4 \\
\hline Vestir metade superior & 3 & 4 & 3 & 4 & 5 & 7 & 3 & 4 \\
\hline Vestir metade inferior & 2 & 3 & 2 & 3 & 4 & 5 & 3 & 4 \\
\hline \multirow[t]{2}{*}{ Utilização da sanita } & 2 & 3 & 3 & 4 & 4 & 5 & 2 & 4 \\
\hline & \multicolumn{8}{|c|}{ Controlo de Esfíncteres } \\
\hline Bexiga & 2 & 3 & 3 & 5 & 5 & 7 & 5 & 5 \\
\hline \multirow[t]{2}{*}{ Intestino } & 2 & 3 & 3 & 5 & 5 & 7 & 5 & 5 \\
\hline & \multicolumn{8}{|c|}{ Mobilidade e Transferências } \\
\hline $\begin{array}{l}\text { Leito, cadeira, } \\
\text { cadeira de rodas }\end{array}$ & 2 & 3 & 3 & 5 & 3 & 5 & 3 & 4 \\
\hline Sanita & 2 & 3 & 3 & 4 & 3 & 4 & 3 & 4 \\
\hline \multirow[t]{2}{*}{ Banheira, Duche } & 2 & 3 & 3 & 4 & 3 & 4 & 3 & 4 \\
\hline & \multicolumn{8}{|c|}{ Locomoção } \\
\hline Marcha, cadeira de rodas & 2 & 3 & 2 & 4 & 2 & 4 & 3 & 4 \\
\hline \multirow[t]{2}{*}{ Escadas } & 2 & 2 & 2 & 3 & 2 & 3 & 2 & 3 \\
\hline & \multicolumn{8}{|c|}{ Comunicação } \\
\hline Compreensão & 3 & 4 & 4 & 5 & 5 & 5 & 4 & 5 \\
\hline \multirow[t]{2}{*}{ Expressão } & 4 & 4 & 4 & 5 & 7 & 7 & 4 & 5 \\
\hline & \multicolumn{8}{|c|}{ Consciência do mundo exterior } \\
\hline Interação social & 4 & 4 & 5 & 5 & 7 & 7 & 5 & 5 \\
\hline Resolução de problemas & 2 & 2 & 3 & 3 & 5 & 5 & 4 & 4 \\
\hline Memória & 3 & 3 & 3 & 3 & 7 & 7 & 4 & 4 \\
\hline Total & 41 & 58 & 57 & 77 & 80 & 100 & 64 & 79 \\
\hline Resultados & \multicolumn{2}{|c|}{+17} & \multicolumn{2}{|c|}{+20} & \multicolumn{2}{|c|}{+20} & \multicolumn{2}{|c|}{+15} \\
\hline
\end{tabular}

Os resultados apresentados demonstram uma elevada dependência funcional após o AVC. A intervenção estruturada e dirigida ao treino dos autocuidados, treino do equilíbrio e reeducação funcional motora, contribuiu para a diminuição da dependência funcional, capacitando os participantes para níveis de desempenho entre a ajuda mínima a moderada segundo a MIF. 
Nos autocuidados: a alimentação foi o que apresentou menores níveis de dependência em todos os casos e também um dos que apresentou evolução mais favorável. Após a intervenção dois participantes ficaram independentes, um ficou com independência modificada e outro participante com dependência modificada. Na higiene, após a intervenção um participante atingiu a independência completa, dois ficaram com dependência modificada com ajuda mínima e um participante com ajuda moderada. No vestuário o parâmetro "vestir metade inferior" apresentou maiores níveis de dependência, comparativamente com os ganhos obtidos na capacitação para o "vestir a metade superior", no entanto todos os participantes conseguiram evoluir favoravelmente na atividade.

O uso do sanitário após intervenção dois participantes ficaram com dependência modificada e ajuda mínima, um participante com supervisão e outro participante com ajuda moderada. Na mobilidade e transferências, o treino de transferências entre superfícies, a reeducação do reflexo postural, e todos os outros exercícios apresentados no protocolo de intervenção possibilitaram um desempenho com a menor ajuda possível em todos os casos.

A evolução média no equilíbrio foi de 12,75 pontos, três doentes mantiveram-se na categoria Aceitável e um evoluiu para Bom (Fig. 1).

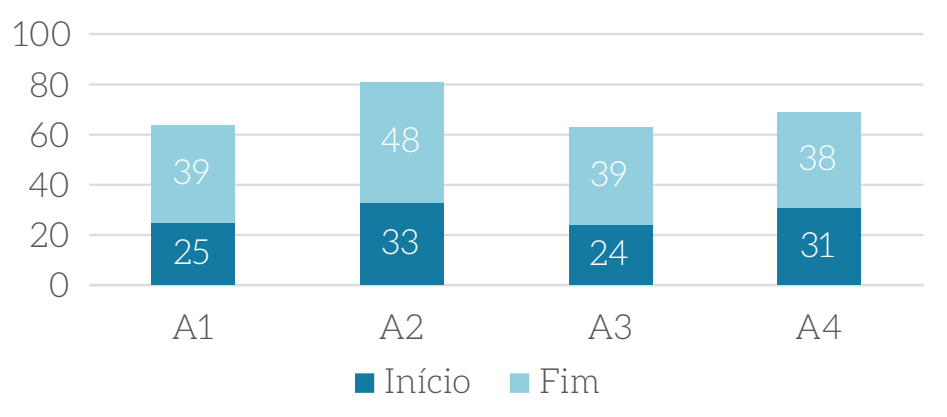

Figura 1 - Avaliação do equilíbrio pré e pós intervenção de enfermagem de reabilitação. 


\section{DISCUSSÃO}

A lesão cerebral adquirida, como o AVC, é uma das causas mais comuns de dependência a longo prazo(1). É uma afeção neurológica comum, quando considerada isoladamente de outras doenças cerebrovasculares, é a quarta principal causa de morte em todo o mundo e a principal razão para a deficiência em contextos comunitários ${ }^{(2)}$.

Os participantes do estudo eram maioritariamente idosos, com idades compreendidas entre os 69 e os 82 anos. Uma participante tinha 57 anos de idade. Os resultados relativos a pessoas idosas com diagnóstico de AVC foram descritos em vários estudos, onde a faixa etária se encontrava acima de 65 anos. 0 aumento da idade está associado a maior mortalidade e maior gravidade resultante do AVC, associado a piores resultados funcionais ${ }^{(10)}$.

Um estudo sobre autonomia/independência no autocuidado verificou que o autocuidado higiene apresentou maiores níveis de dependência na avaliação final. Esta ideia pode ser corroborada pelo facto de os participantes serem idosos com maior índice de dependência que já detêm dificuldades na realização de determinadas tarefas das suas AVD como conseguir lavar/secar o corpo, entre outras ${ }^{(11)}$. Os resultados podem assim ser explicados tendo em conta as características das pessoas.

A capacitação dos doentes para os autocuidados é eficaz no incremento da funcionalidade ${ }^{(12)}$ tal como foi verificado nos casos estudados. $O$ ensino e o treino dos autocuidados segundo um protocolo estruturado revelaram-se eficazes, com ganhos funcionais avaliados pela MIF.

O estudo de Aries et al refere que atividades fora do leito, como sentar-se e ficar de pé, estar de pé, caminhar até ao sanitário, realizar transferências no sanitário, são potenciadores de uma participação ativa da pessoa no seu processo de reabilitação e aumentam o seu bem-estar(3).

As atividades de transferência e de movimentação no leito conjugam estratégias de força, coordenação de movimentos e equilíbrio e os resultados demonstram a evolução favorável obtida por todos os doentes não só na realização do autocuidado como também nos ganhos no equilíbrio. Uma revisão da literatura constatou a importância que a prática e a repetição de movimentos sejam incorporados às atividades funcionais e/ou $\mathrm{AVD}^{(3)}$. A avaliação da marcha, o treino de marcha com a realização de treinos de curta duração várias vezes durante o turno e a reaquisição do padrão automático perdido, tendo em conta o dispêndio de energia, são intervenções com benefício para a pessoa. Uma adequada intervenção e abordagem denominada como "mobilização precoce" reduz significativamente complicações associadas ao tempo de permanência no leito(3). A evolução positiva a este ní- 
vel foi um fator facilitador na habilidade para a execução dos autocuidados. O processo de recuperação e adaptação a uma incapacidade, permite à pessoa ajustar-se a uma nova vida e a um corpo alterado(13), através de um plano individualizado e estruturado capacitam-se pessoas com necessidades especiais e limitações da funcionalidade.

\section{CONCLUSÃO}

Todos os participantes obtiveram um aumento da sua funcionalidade, maximizando o seu desempenho motor e cardiorrespiratório de acordo com a especificidade de cada pessoa, a sua tolerância ao esforço e a sua capacidade física, fatores tidos em conta na intensidade das atividades. Os resultados obtidos confirmam a importância da intervenção de enfermagem de reabilitação, reafirmam as competências na área da especialidade e indiciam a importância de modelos de cuidados reabilitação com inicio na fase aguda. As limitações ao estudo decorreram do tempo de contacto com cada participante e à inexistência de homogeneidade nos casos estudados.

Declaração de interesses/Declaration of Interests

Estudo realizado no âmbito do Estágio final do Curso de Mestrado em Enfermagem na área de especialização de enfermagem de reabilitação.

\section{Responsabilidades Éticas}

Conflitos de Interesse: Os autores declaram a inexistência de conflitos de interesse na realização do presente trabalho.

Fontes de Financiamento: Não existiram fontes externas de financiamento para a realização deste artigo.

Confidencialidade dos Dados: Os autores declaram ter seguido os protocolos da sua instituição acerca da publicação dos dados de doentes.

Proteção de Pessoas e Animais: Os autores declaram que os procedimentos seguidos estavam de acordo com os regulamentos estabelecidos pelos responsáveis da Comissão de Investigação Clínica e Ética e de acordo com a Declaração de Helsínquia da Associação Médica Mundial.

Proveniência e Revisão por Pares: Não comissionado; revisão externa por pares.

\section{Ethical Disclosures}

Conflicts of interest: The authors have no conflicts of interest to declare.

Financing Support: This work has not received any contribution, grant or scholarship.

Confidentiality of Data: The authors declare that they have followed the protocols of their work center on the publication of data from patients.

Protection of Human and Animal Subjects: The authors declare that the procedures followed were in accordance with the regulations of the relevant clinical research ethics committee and with those of the Code of Ethics of the World Medical Association (Declaration of Helsinki).

Provenance and Peer Review: Not commissioned; externally peer reviewed. 


\section{REFERÊNCIAS}

1. Bender A, Adrion C, Fischer L, Huber M, Jawny K, Straube A, et al. Long-term rehabilitation in patients with acquired brain injury: A randomized controlled trial of an intensive, participation-focused outpatient treatment program. Dtsch Arztebl Int. 2016;113:634.

2. Klinke ME, Hafsteinsdottir TB, Hjaltason H, Jonsdottir H. Ward-based interventions for patients with hemispatial neglect in stroke rehabilitation: a systematic literature review. Int J Nurs Stud. 2015;52:1375-403.

3. Aries A, Hunter SM. Optimising rehabilitation potential after stroke: a 24-hour interdisciplinary approach. Br J Neurosci Nurs. 2014;10:268-73.

4. Ahmed N, Audebert H, Turc G, Cordonnier C, Christensen H, Sacco S, et al. Consensus statements and recommendations from yhe ESO-Karolinska Stroke Update Conference, Stockholm 11-13 November 2018. Eur Stroke J. 2020;4:307-17.

5. Gagnier JJ, Kienle G, Altman DG, Moher D, Sox H, Riley D, et al. The CARE Guidelines: Consensus-based Clinical Case Reporting Guideline Development. Glob Adv Health Med. 2013;2:38-43. doi:10.7453/gahmj.2013.008

6. Ordem dos Enfermeiros. CIPE, Versão 2015 - Classificação Internacional para a Prática de Enfermagem. Loures: Lusodidacta, 2016.

7. Direção Geral da Saúde. Acidente Vascular Cerebral: Prescrição de medicina física e de reabilitação - Norma n. o 54/2011 de 27/12/2011. Lisboa: DGS; 2011.

8. Mesa do Colégio da Especialidade de Enfermagem de Reabilitação. Instrumentos de recolha de dados para a documentação dos Cuidados Especializados em Enfermagem de Reabilitação. Lisboa: Ordem dos Enfermeiros; 2016.

9. Silva Ad, Almeida GJ, Cassilhas RC, Cohen M, Peccin MS, Tufik S, et al. Equilíbrio, coordenação e agilidade de idosos submetidos à prática de exercícios físicos resistidos. Rev Bras Edu Fís Esp. 2008;14:88-93.

10. O'Brien SR, Xue Y. Inpatient rehabilitation outcomes in patients with stroke aged 85 years or older. Phys Ther. 2016;96:1381-8.

11. Couto G. Autonomia/Independência no Autocuidado Sensibilidade aos Cuidados de Enfermagem de Reabilitação [Dissertação de Mestrado]. Porto: Escola Superior de Saúde do Porto; 2012. 
CUIDADOS PRECOCES NA REABILITAÇÃO DA FUNCIONALIDADE

12. Reis G, Bule MJ. Capacitação e Atividade de Vida. In: Marques-Vieira C, Sousa L, editores. Cuidados de Enfermagem de Reabilitação ao longo da Vida. Loures: Lusodidacta; 2017. p.57-66.

13. Menoita E. Reabilitar a Pessoa Idosa com AVC: Contributos para um envelhecer resiliente. Loures: Lusociência; 2014. 